\title{
Model of electronic states at the $\mathrm{Si}_{-} \mathrm{SiO}_{2}$ interface
}

\author{
A. S. Carrico, ${ }^{*}$ R. J. Elliott, and R. A. Barrio ${ }^{\dagger}$ \\ Department of Theoretical Physics, Oxford University, 1 Keble Road, Oxford OX1 3NP United Kingdom
}

(Received 10 January 1986)

\begin{abstract}
The electronic properties of the interface between crystalline $\mathrm{Si}$ and its amorphous oxide $\mathrm{SiO}_{2}$ have been studied within the tight-binding approximation by saturating the dangling bonds in three different surfaces (111), (110), and (100) of a semi-infinite $\mathrm{Si}$ crystal with $\mathrm{SiO}_{2}$ Bethe lattices. The localized states due to isolated dangling bonds in the interfaces $\left(P_{b}\right.$ centers) are found to be roughly at the same energy, in the Si gap for the (111) and (110) cases, but the (100) interface differs substantially from the other two. It is also found that the value of the site-energy interaction parameter on the Si defect site is important in determining the energy of the defect state.
\end{abstract}

\section{INTRODUCTION}

A basic understanding of the nature of interface electron traps and defects in the $\mathrm{Si}-\mathrm{SiO}_{2}$ system is important for the technological challenge of fabricating metaloxide-semiconductor (MOS) devices on the submicrometer scale. Unfortunately since such defects and traps modify substantially the performance of these small-scale devices the most important states are the ones which occur around the Si gap. These have been investigated by $C-V$ measurements ${ }^{1}$ and deep-level transient spectroscopy. ${ }^{2}$ The densities of states (DOS's) obtained by properly deconvoluting the experimental data agree in both sorts of experiments and consist, around the Si gap, of exponential tails on each end of the gap from localized states in the interface and two clearly noticeable peaks at around 0.26 and $0.83 \mathrm{eV}$ from the valence-band edge. The two welldefined peaks are believed to be the two charge states of a three-bonded $\mathrm{Si}$ (a $\mathrm{Si}$ with a dangling bond) at the interface $\left(P_{b}\right.$ center $){ }^{3}$ This interpretation has been corroborated by electron-spin-resonance measurements ${ }^{4}$ and by careful examination of different crystallographic orientations for the interface. A recent review by Poindexter et al. ${ }^{5}$ contains all the relevant experimental information available so far.

From the theoretical side, there have been various attempts to model the interface. Most of them concentrate on the highly symmetric Si-(111) surface. There are two recent papers which emphasize two different points of view:

(1) A tight-binding approach by Sakurai and Sugano ${ }^{6}$ in which a semi-infinite $\mathrm{Si}$ crystal with a (111) surface is saturated with a Bethe lattice in various "wrong" ways producing $\mathrm{Si}-\mathrm{Si}$ bonds, $\mathrm{Si}$ dangling bonds, and weak $\mathrm{Si}-\mathrm{O}$ bonds at the interface. By linearly varying the tightbinding interactions with the distance between atoms, they examine the variation of the position of the defect state in the gap. It is seen that the "weak" $\mathrm{Si}-\mathrm{Si}$ bond moves towards the valence band, while the "weak" $\mathrm{Si}-\mathrm{O}$ bond produces a shift towards the conduction band; and in this way it is claimed that the exponential tailing of the band edges is produced in the gap. However, the arbitrary variation of parameters which is assumed severely restrains the important conclusions of the theory.

(2) The other is a finite-cluster calculation by Edwards ${ }^{7}$ that is more concerned with the single $P_{b}$-center peaks. The electron-electron correlations are introduced in a way similar to the complete-neglect-of-differential-overlap approach and the full Hamiltonian is diagonalized for the cluster. As well as the eigenvalues for the defect state, the total energy is calculated. This allows the relaxed position of the defect to be found in the different charge states. Correlation energies and spin densities are also found and they agree well with experiment. Unfortunately, this calculation suffers from the usual drawbacks deriving from the finite size of the clusters used.

It is the purpose of this paper to report a theory that removes some of the constraints pointed out above. In Sec. II we introduce the basic concepts of the theory and give the solutions for the three free crystalline surfaces to be studied, (111), (110), and (100). In Sec. III we present the solutions of a $\mathrm{SiO}_{2}$ Bethe lattice and build up perfect interfaces. In Sec. IV we discuss a $P_{b}$ center in the three perfect interfaces and the local DOS at the defect site.

\section{PERFECT SURFACES}

A. The tight-binding model

When $\mathrm{Si}$ atoms form a solid, the valence electrons rearrange to form four $s p^{3}$ hybrid orbitals directed towards the vertices of a tetrahedron. The tight-binding approximation uses a Hamiltonian with a basis of four $s p^{3}$ orbitals localized on each atom. Only interactions between nearest neighbors are considered. In Fig. 1 we show the

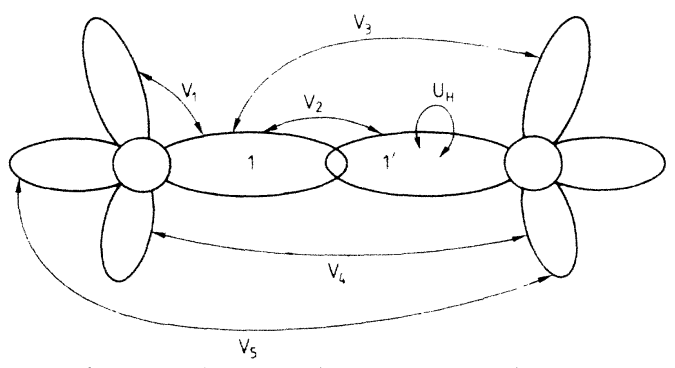

FIG. 1. Diagram showing the nearest-neighbor tight-binding interactions between $s p^{3}$ hybrid orbitals in Si. 
nature of all the interactions parametrized. With this notation the matrix elements of the Hamiltonian confined to the site of a given atom form the $4 \times 4$ site-energy matrix,

$$
A=\left(\begin{array}{cccc}
U_{H} & V_{1} & V_{1} & V_{1} \\
V_{1} & U_{H} & V_{1} & V_{1} \\
V_{1} & V_{1} & U_{H} & V_{1} \\
V_{1} & V_{1} & V_{1} & U_{H}
\end{array}\right),
$$

and the matrix for the interaction between two neighbors along bond 1-1' in Fig. 1 is

$$
B=\left(\begin{array}{llll}
V_{2} & V_{3} & V_{3} & V_{3} \\
V_{3} & V_{5} & V_{4} & V_{4} \\
V_{3} & V_{4} & V_{5} & V_{4} \\
V_{3} & V_{4} & V_{4} & V_{5}
\end{array}\right) .
$$

We are interested in studying semi-infinite crystals with the diamond structure and with a single surface. The presence of the surface breaks the translational symmetry of the lattice in the direction perpendicular to the surface; therefore, it is convenient to use a representation in which this fact is taken into account,

$$
\phi_{n}^{j}(\mathbf{K})=\frac{1}{\sqrt{N}} \sum_{\mathbf{R}_{n}} e^{i \mathbf{K} \cdot \mathbf{R}_{n}} \phi^{j}\left(\mathbf{R}_{n}\right),
$$

where $\phi^{j}\left(\mathbf{R}_{n}\right)$ is one of the $j=1, \ldots, 4$ orbitals centered on the atom situated at $\mathbf{R}_{n}$ in the plane $n$ from the surface. The vector $\mathbf{K}$ is parallel to the surface and $N$ is the normalizing factor per plane. In this representation we can define an energy-dependent Green's function ${ }^{8}$ that obeys the equation of motion,

$$
\begin{aligned}
G_{K}(n, m)(E I-A)= & \delta(n, m) I+G_{K}(n, m+1) H_{K}(m+1, m) \\
& +G_{K}(n, m-1) H_{K}(m-1, m) .
\end{aligned}
$$

All quantities in (4) are $4 \times 4$ matrices whose elements are written in the representation (3); thus, for instance,

$$
G_{K}^{j j^{\prime}}(n, m)=\left\langle\phi_{n}^{j}(\mathbf{K})|G| \phi_{m}^{j^{\prime}}(\mathbf{K})\right\rangle \text {. }
$$

In order to solve (4), one needs to specify the plane-toplane interactions $H(n, n \pm 1)$, which depend on the particular crystallographic plane of the structure and hence give different detailed solutions for the three surfaces considered.

\section{B. (111) perfect surface}

In this case we have two different bondings between planes. There are neighbor planes that are linked by one bond per atom perpendicular to the surface and planes that are linked by the remaining three bonds. We take the surface at $n=0$ to have one dangling bond per atom. Then,

$$
H_{K}(n, n+1)=\left\{\begin{array}{l}
B \text { if } n \text { is odd }, \\
C^{*}(\mathbf{K}) \text { if } n=0, \text { even },
\end{array}\right.
$$

where $B$ is given by (2) and

$$
C(\mathbf{K})=\sum_{j=2}^{4} e^{i \mathbf{K} \cdot \mathbf{R}_{j}} S_{j}^{-1} B S_{j},
$$

where $S_{j}$ is a $4 \times 4$ matrix that transforms the hybrid 1 into the hybrid $j$ and $\mathbf{R}_{j}$ are the relative positions of the nearest atoms in adjacent planes. Following Falicov and Ynduráin, ${ }^{9}$ these are

$$
\mathbf{R}_{2}=\boldsymbol{\sigma}, \quad \mathbf{R}_{3}=\boldsymbol{\sigma}+\mathbf{t}_{2}, \quad \text { and } \mathbf{R}_{4}=\boldsymbol{\sigma}+\mathbf{t}_{1}+\mathbf{t}_{2} \text {, }
$$

where $\sigma=-(a / \sqrt{6})(0,1,0), \quad t_{1}=(a / \sqrt{2})(1,0,0), \quad$ and $\mathrm{t}_{2}=(a / 2 \sqrt{2})(-1, \sqrt{3}, 0)$.

It is clear that

$$
H_{K}(n, n-1)=\left\{\begin{array}{l}
B \text { if } n \text { even }, \\
C(\mathbf{K}) \text { if } n \text { odd },
\end{array}\right.
$$

and $H_{K}(-1,0)=H_{K}(0,-1)=0$.

Equation (4) is solved by defining the following transfer matrices,

$$
\begin{aligned}
& G_{K}(n, 2 m+1)=G_{K}(n, 2 m) T_{1}(\mathbf{K}), \\
& G_{K}(n, 2 m+2)=G_{K}(n, 2 m+1) T_{2}(\mathbf{K}), \\
& G_{K}(n, 2 m-1)=G_{K}(n, 2 m) Q_{1}(\mathbf{K}), \\
& G_{K}(n, 2 m)=G_{K}(n, 2 m+1) Q_{2}(\mathbf{K}),
\end{aligned}
$$

that obey the equations

$$
\begin{aligned}
& T_{1}(\mathbf{K})=C^{*}(\mathbf{K})\left[E I-A-T_{2}(\mathbf{K}) B\right]^{-1}, \\
& T_{2}(\mathbf{K})=B\left[E I-A-T_{1}(\mathbf{K}) C(\mathbf{K})\right]^{-1}, \\
& Q_{1}(\mathbf{K})=B\left[E I-A-Q_{2}(\mathbf{K}) C^{*}(\mathbf{K})\right]^{-1}, \\
& Q_{2}(\mathbf{K})=C(\mathbf{K})\left[E I-A-Q_{1}(\mathbf{K}) B\right]^{-1},
\end{aligned}
$$

which can be solved iteratively. All correlations between planes for a given $\mathbf{K}$ can be obtained with (10) and (4). Local Green's functions and densities of states are obtained by summing over the $K$ space in the twodimensional Brillouin zone $(K-\mathrm{Bz})$. The numerical effort of performing the sum was minimized by choosing a network of points in the irreducible part of the Brillouin zone in the way described by Cunningham. ${ }^{10}$

\section{C. (110) perfect surface}

In this case each atom has two bonds connecting it to atoms in a plane parallel to the surface, and the remaining two connect it to the adjacent planes. There are two inequivalent positions in the two-dimensional unit cell: therefore it is convenient to consider $8 \times 8$ matrices in $\mathrm{Eq}$. (4). The coupling between planes is

$$
H_{K}(n, n+1)=\left(\begin{array}{cc}
0 & B e^{i \mathbf{K} \cdot \mathbf{R}_{1}} \\
S_{2}^{-1} B S_{2} e^{i \mathbf{K} \cdot \mathbf{R}_{2}} & 0
\end{array}\right)
$$

and

$$
H_{K}(n, n-1)=\left(\begin{array}{cc}
0 & S_{2}^{-1} B S_{2} e^{-i \mathbf{K} \cdot \mathbf{R}_{2}} \\
B e^{-i \mathbf{K} \cdot \mathbf{R}_{1}} & 0
\end{array}\right),
$$


and the intraplane coupling is

$$
H_{K}(n, n)=\left(\begin{array}{cc}
A & V \\
V^{*} & A
\end{array}\right)
$$

where

$$
V=S_{3}^{-1} B S_{3} e^{i \mathbf{K} \cdot \mathbf{R}_{3}}+S_{4}^{-1} B S_{4} e^{i \mathbf{K} \cdot \mathbf{R}_{4}} .
$$

The solution is found by defining

$$
G_{K}(n, m+1)=G_{K}(n, m) T(\mathbf{K})
$$

that satisfies

$$
\begin{aligned}
T(\mathbf{K})=[ & E I-H_{K}(n, n) \\
& \left.-T(\mathbf{K}) H_{K}(n, n-1)\right]^{-1} H_{K}(n, n+1) .
\end{aligned}
$$

The local Green's function at the surface is given by

$G(0,0)=\sum_{K=\mathrm{BZ}}\left[E I-H_{K}(n, n)-T(\mathbf{K}) H_{K}(n, n-1)\right]^{-1}$,

and the density of surface states is

$$
\rho_{0}(E)=\frac{-1}{\pi} \operatorname{Im} \operatorname{Tr} G(0,0)
$$

\section{D. (100) perfect surface}

In this case the interaction between adjacent planes parallel to the surface is by two bonds per atom. These interactions alternate because the pair of bonds linking two given planes form a plane perpendicular to the one formed by the other pair of bonds. Therefore the mathematical treatment is rather similar to the (111) case, the only difference being substitution of $B \rightarrow B^{\prime}(\mathbf{K})$ and $C(\mathbf{K}) \rightarrow C^{\prime}(\mathbf{K})$, where

$$
B^{\prime}(\mathbf{K})=\sum_{j=3,4} S_{j}^{-1} B S_{j} e^{i \mathbf{K} \cdot \mathbf{R}_{j}}
$$

and

$$
C^{\prime}(\mathbf{K})=\sum_{j=1,2} S_{j}^{-1} B S_{j} e^{i \mathbf{K} \cdot \mathbf{R}_{j}}
$$

\section{E. Results}

In Fig. 2 we show the density of states for the valence band in bulk Si obtained with $64 \times 8$ points in the Brillouin zone, using the parameters given in Table I. These give an accurate reproduction of the valence band and the gap (cf., for example, Ref. 11), but they predict a conduction band which is narrower than that found experimentally. Further orbitals must be added to the tight-binding scheme to give these bands accurately as well. ${ }^{12}$ In Fig. 3 we show the results for the perfect surfaces around the re-

TABLE I. Parameters used for the Si crystal (in eV).

\begin{tabular}{cccccc}
\hline \hline$U_{H}$ & $V_{1}$ & $V_{2}$ & $V_{3}$ & $V_{4}$ & $V_{5}$ \\
\hline-0.885 & -1.435 & -3.531 & -0.541 & -0.261 & 0.549 \\
\hline \hline
\end{tabular}

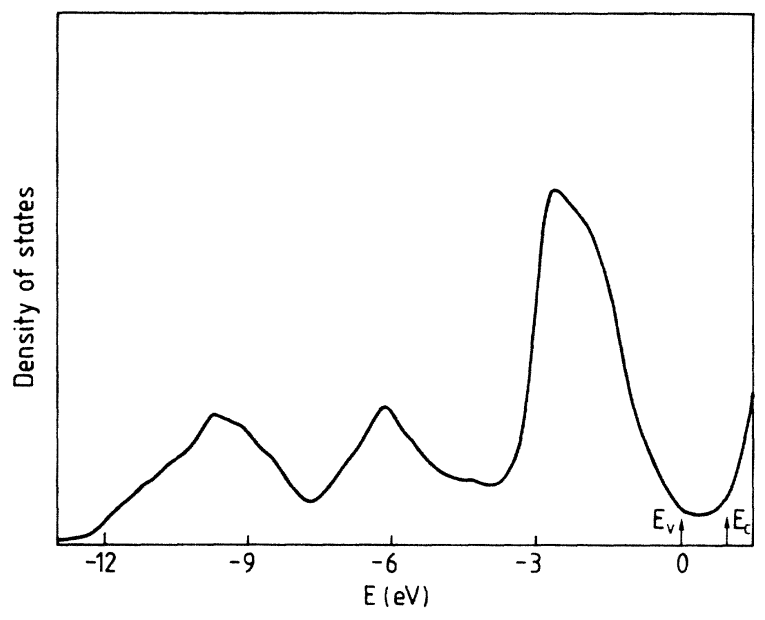

FIG. 2. Density of valence-band states for bulk $\mathrm{Si}$ in the tight-binding model.

gion of the gap. In the case of the (111) surface we obtain a surface band that extends from the middle of the gap to the valence band, with a width of $\sim 0.8 \mathrm{eV}$, in agreement with previous calculations. ${ }^{13}$ We notice that these surface states leak into the backbonds of the surface atoms, and this effect is enhanced if the surface is allowed to relax. ${ }^{14}$

In the (110) case there are two dangling bond bands, one for each type of atom in the unit cell. These bands are wider than in the previous case due to the direct interaction between atoms in the surface plane. The (100) surface presents also two bands around the gap region due to the two dangling bonds per atom in the surface.

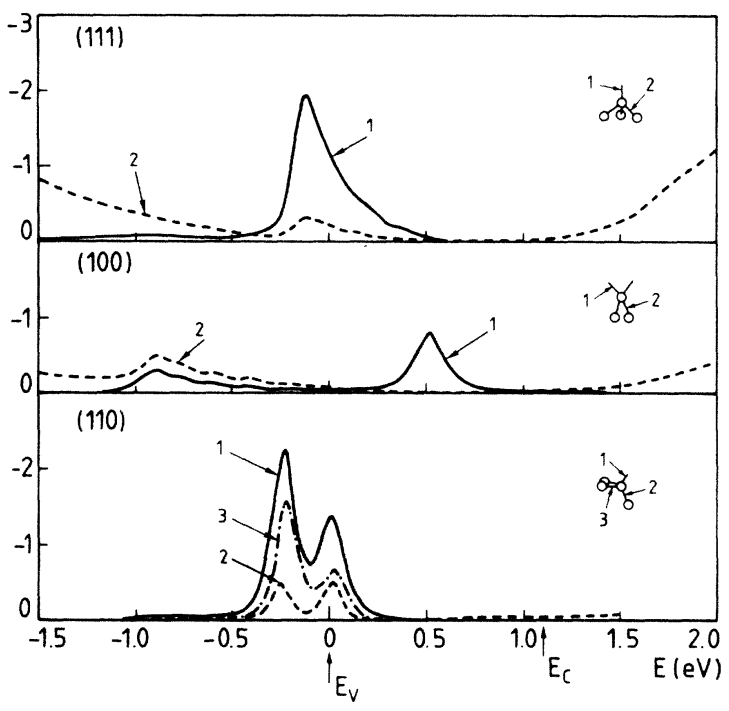

FIG. 3. Local density of states on the surface orbitals of three different crystallographic orientations. The orbitals that correspond to each curve are shown in the insets. The solid lines refer to dangling bonds which are largely localized states in the surface. The dashed lines refer to backbonds connected to the lattice. They are multiplied by a factor of 7.5 relative to the solid lines showing finite but weak contributions in the surface bands within the gap. 


\section{PERFECT INTERFACES}

\section{A. Bethe-lattice model of $\mathrm{SiO}_{2}$}

In order to model a perfect interface between the $\mathrm{Si}$ crystal and vitreous $\mathrm{SiO}_{2}$, we have simulated the latter substance by a Bethe lattice. The $\mathrm{SiO}_{2}$ Bethe lattice has been constructed by Laughlin et al. ${ }^{15}$ and more recently used for interfaces of the (111) type by Martinez and Ynduráin. ${ }^{16}$ However, we think that it is necessary to give a brief account here of the procedure followed. The basic unit of $\mathrm{SiO}_{2}$ is a $\mathrm{Si}$ atom surrounded by four oxygens in the corners of a tetrahedron. These tetrahedra are joined by oxygen bridges that conserve the $\mathrm{Si}-\mathrm{O}-\mathrm{Si}$ angle pretty well. The dihedral angles between adjacent tetrahedra are believed to be largely random, but if one neglects oxygen $p p \pi$ interactions, the Hamiltonian does not depend on the dihedral angles. With these assumptions the problems can be mapped into an effective Si lattice and the Si-Si interactions are the same as (1) and (2) with effective interactions $U_{H}^{\text {eff }}$ and $V_{i}^{\text {eff }}$ which depend on the energy and contain the oxygen coordinates. The Si-O interactions are illustrated in Fig. 4. With these parameters the expressions for the effective interactions are

$$
\begin{aligned}
& U_{H}^{\text {eff }}=U_{H 0}+\frac{3\left(T^{\prime}\right)^{2}+T^{2}}{E-E_{p}}+\frac{3\left(\tau^{\prime}\right)^{2}+\tau^{2}}{E-E_{s}}, \\
& V_{1}^{\text {eff }}=V_{10}+\frac{T T^{\prime}+\left(T^{\prime}\right)^{2}}{E-E_{p}}+\frac{\tau \tau^{\prime}+\left(\tau^{\prime}\right)^{2}}{E-E_{s}}, \\
& V_{2}^{\text {eff }}=\frac{T^{2} \cos \theta}{E-E_{p}}+\frac{\tau^{2}}{E-E_{s}}, \\
& V_{3}^{\text {eff }}=\frac{T T^{\prime} \cos \theta}{E-E_{p}}+\frac{\tau \tau^{\prime}}{E-E_{s}}, \\
& V_{4}^{\text {eff }}=V_{5}^{\text {eff }}=\frac{\left(T^{\prime}\right)^{2} \cos \theta}{E-E_{p}}+\frac{\left(\tau^{\prime}\right)^{2}}{E-E_{s}},
\end{aligned}
$$

where $E_{p}$ and $E_{s}$ are the atomic levels for the oxygen $p$ and $s$ orbitals, respectively, and $V_{10}$ and $U_{H 0}$ have the same meaning as $V_{1}$ and $U_{H}$ in Eqs. (19), but for a $\mathrm{Si}$ atom surrounded by four oxygen atoms. In the case of the Bethe lattice the equations of motion are of the type

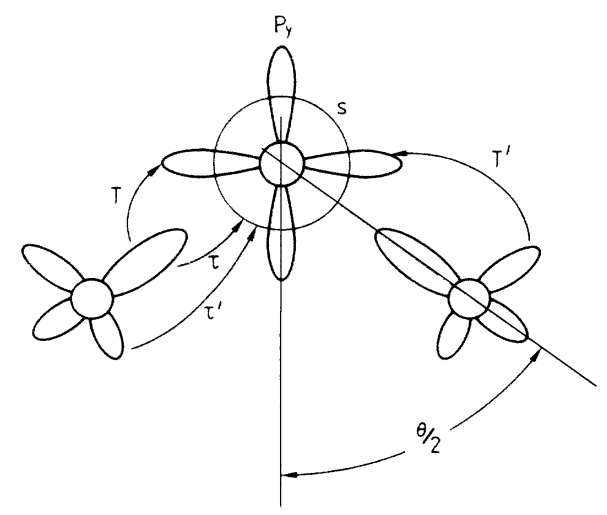

FIG. 4. Diagram showing the Si-O tight-binding interactions considered to construct a $\mathrm{SiO}_{2}$ network. The angle $\theta$ was taken to be fixed at $144^{\circ}$.
(4) with the shell-generation number taking the role of a plane. (Obviously there is no $K$ vector in this case.) The transfer matrix $\Sigma_{1}$ for the Bethe lattice along the bond 1 obeys the equation

$$
\Sigma_{1}=\left[E I-A^{\mathrm{eff}}-\sum_{j \neq 1)} S_{j}^{-1} \Sigma_{1} B^{\mathrm{eff}} S_{j}\right]^{-1} B^{\mathrm{eff}} .
$$

The parameters appropriate for a $\mathrm{SiO}_{2}$ Bethe lattice are given in Table II and the energy bands derived from them are shown in Fig. 5.

\section{B. Interface}

To describe an interface between $\mathrm{SiO}_{2}$ and $c$-Si, an effective medium, with properties governed by (20), is attached to each dangling bond in the crystal surface. For example, the Green's function in the (111) interface is

$$
G_{K}(0,0)=\left[E I-A^{\prime}-T_{1} C^{*}(\mathbf{K})-\Sigma_{1} B^{\text {eff }}\right]^{-1} .
$$

The site-energy matrix $A^{\prime}$ in the surface layer will differ from that in the perfect crystal and from that in $\mathrm{SiO}_{2}$ The simplest assumption is to interpolate between the values $U_{H}$ and $U_{H 0}$ according to the number of oxygen neighbors [Eq. (27)]. However, self-consistent calculations using the Hartree-Fock method described in the accompanying paper ${ }^{17}$ suggest that $U_{H}^{\prime}$ may be significantly reduced as electrons are attracted towards the oxygen by the bond ionicity.

In the other surfaces, the method follows similarly, except that the interactions between the dangling bonds and the Bethe lattices have to be properly oriented. For instance, the Green's function at the (100) interface will be

$$
\begin{aligned}
G_{K}(0,0)=[ & E I-A^{2}-T_{1} C^{\prime}(\mathbf{K}) \\
& \left.-S_{3}^{-1} \Sigma_{1} B^{\mathrm{eff}} S_{3}-S_{4}^{-1} \Sigma_{1} B^{\mathrm{eff}} S_{4}\right]^{-1},
\end{aligned}
$$

where the site-energy matrix $A^{2}$ has been properly renor-

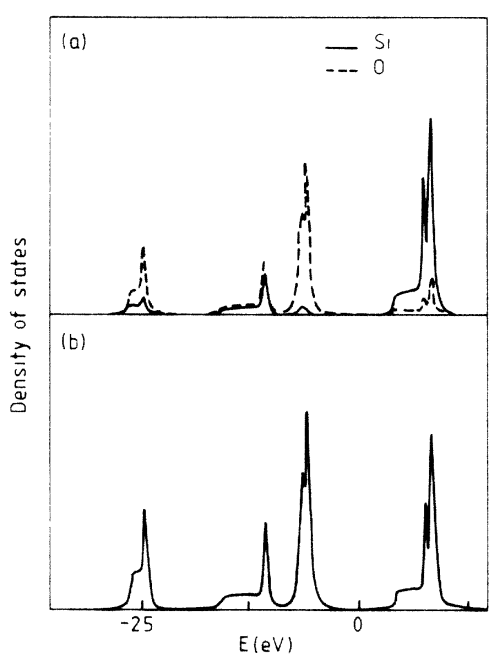

FIG. 5. (a) Densities of states in a $\mathrm{SiO}_{2}$ Bethe lattice: on a $\mathrm{Si}$ site ( - ) and on an oxygen site ( $-\ldots)$; (b) total density of states. The parameters used are shown in Table II and were chosen to fit the position of the $\mathrm{Si}$ gap in the $\mathrm{Si}_{-} \mathrm{SiO}_{2}$ interface. 
TABLE II. Parameters used for the $\mathrm{SiO}_{2}$ Bethe lattice (in eV).

\begin{tabular}{ccccccccc}
\hline \hline$U_{H 0}$ & $V_{10}$ & $E_{s}$ & $E_{p}$ & $T$ & $\tau$ & $T^{\prime}$ & $\tau^{\prime}$ & $\theta(\mathrm{deg})$ \\
\hline 2.167 & -1.6 & -20.83 & -5.63 & -7.0 & -7.2 & -0.65 & -0.2 & 144 \\
\hline \hline
\end{tabular}

malized to take into account the fact that the Si atom is connected to two oxygen atoms, through bonds 3 and 4 [Eq. (27)].

The (110) interface is constructed in the same way, except that the matrices are now $8 \times 8$. The local Green's function at the silicon atom in the interface has the form (16) with the addition in the denominator of

$$
\Sigma^{\prime}=\left(\begin{array}{cc}
S_{2}^{-1} \Sigma_{1} B^{\mathrm{eff}} S_{2} & 0 \\
0 & S_{2}^{-1} \Sigma_{1} B^{\text {eff }} S_{1}
\end{array}\right),
$$

and again an appropriate modification of $U_{H}$ and $V_{1}$.

The local densities at the perfect interface are obtained with the Brillouin sum of (21), (22), and (16), plus (23) using (17). No states in the Si gap were obtained in any interface if the site energies were interpolated as described, and none were obtained if $U_{H}^{\prime}$ is reduced from -0.885 as in Table $I$ to -2.37 , as suggested by the self-consistent calculations. If $U_{H}^{\prime}$ is increased by a large amount $(3 \mathrm{eV}$ was used in a calculation), states do appear in the gap, but this does not appear to correspond. to the physical situation. Other tight-binding calculations of defects in $\mathrm{SiO}_{2}$ (Ref. 18) use the bulk Si value for the $U_{H}$ when there is a $\mathrm{Si}-\mathrm{Si}$ bond and $U_{H}+\Delta=U_{H 0}$ when there is a $\mathrm{Si}-\mathrm{O}-\mathrm{Si}$ bond.

\section{IV. $P_{b}$ CENTERS}

We now consider an isolated $P_{b}$ center in an otherwise perfect interface. The perturbation consists in cutting the interactions between a single $\mathrm{Si}$ atom and its attached $\mathrm{SiO}_{2}$ Bethe lattice. In order to do so we have to work in real space and let two atoms of the perfect system be affected by the perturbation, which means that the impurity space is $8 \times 8$.

We shall use Dyson's equation to find the perturbed Green's function

$$
G=G^{0}+G^{0} U G
$$

where the unperturbed Green's function is

$$
G^{0}=\left[\begin{array}{ll}
G(0,0) & G(0,1) \\
G(1,0) & G(1,1)
\end{array}\right],
$$

each entry being a $4 \times 4$ matrix of the type (16); the offdiagonal elements $G(0,1)$ can be found for each interface using the appropriate transfer matrices defined in Secs. II and III.

The perturbation must contain the renormalized site energies for the atoms with the dangling bond in the Si crystal (site 0) and the first Si atom in the Bethe lattice (site 1); for instance, for a $P_{b}$ center in a (111) interface,

$$
U=\left(\begin{array}{cc}
A-A^{1} & -B^{\text {eff }} \\
-B^{\text {eff }} & A^{\text {eff }}-A^{3}
\end{array}\right)
$$

where $A^{j}$ is the self-energy of a $\mathrm{Si}$ atom with $j$ oxygen bonds. A linear interpolation from the bulk values in $\mathbf{S i}$ and $\mathrm{SiO}_{2}$ gives a renormalized site-energy matrix

$$
A^{j}=\frac{1}{4}\left[(4-j) A+j A^{\mathrm{eff}}\right] \text {. }
$$

A self-consistent calculation of the energy $A^{1}$ at the termination of a Si Bethe lattice has been made in the adjoining paper. This reduces $U_{H}$ by $0.9 \mathrm{eV}$ from the usual value. In a simple model of a $P_{b}$ center with double electron occupancy, the extra repulsion increases the site energy by $1.2 \mathrm{eV}$. To illustrate this effect we make calculations with varying values of this parameter.

The local densities of states in each orbital of the defect atom are the first four diagonal elements of Eq. (24). In all three interfaces the form of the equations is preserved, although the geometry considered is substantially different. In Fig. 6 we show the $P_{b}$ center in the three interfaces. We notice that the local environment of the defect is very similar for the (111) and (110) interfaces, but different for the (100). There could be two types of $P_{b}$ center in this latter case; here we only considered the one marked $P_{b}$ since the other involves considerable rearrangement of the interface.

In Fig. 7 we show the $P_{b}$-center local response calculated using the interpolated value of the site energies. The dangling-bond response is found to be near the valenceband edge in the (111) and (110) cases, in agreement with

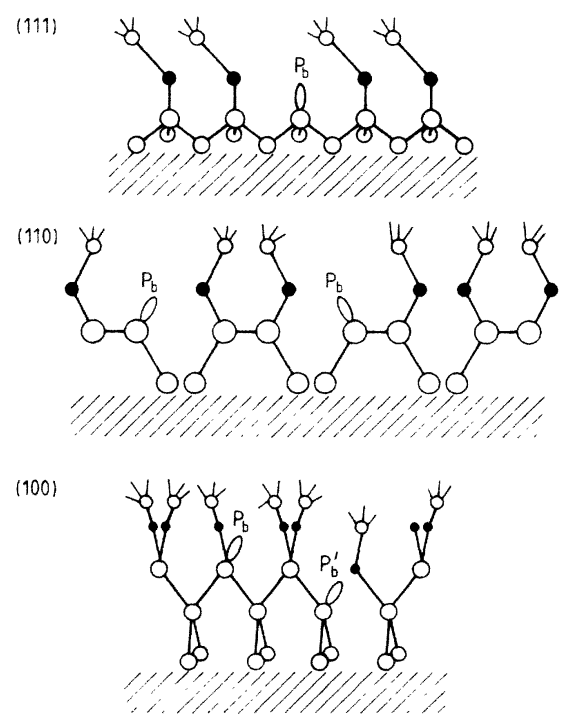

FIG. 6. Diagram showing the local environment of a $P_{b}$ center in the three different interfaces considered. Note that the two $P_{b}$ centers shown in the (110) interface are equivalent and similar to the (111) $P_{b}$ center. The (100) interface has two inequivalent centers. The one marked $P_{b}^{\prime}$ has a different environment and is not considered here. 


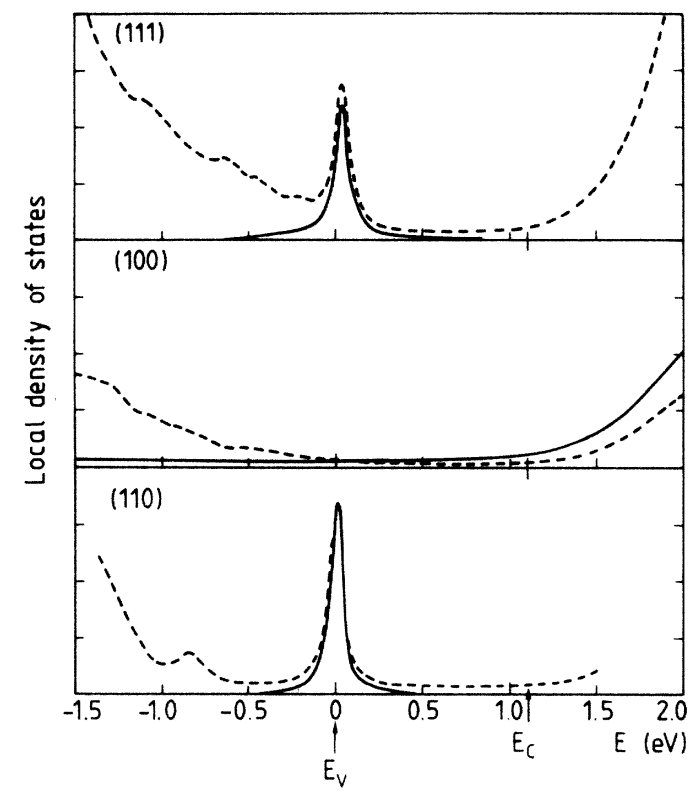

FIG. 7. Local DOS in a $P_{b}$ center for the three types of interfaces considered. The solid curve shows the dangling-bond response and the dotted curve the backbond response amplified 5 times.

numerical calculations in clusters, ${ }^{7}$ but in the $(100)$ case it is found to be practically immersed in the conduction band. This, we believe, is an important point, since it could be explanation of the well-known fact that this interface gives very few traps in the Si gap. ${ }^{5}$ The local densities in the backbonds belonging to the same defect atom are shown in Fig. 7. We notice that the impurity state leaks into the backbonds, and therefore there must be some relaxation of the defect atoms. These results depend only slightly on the nature of the site energies used in the interface.

The energies of the states in the gap are not comparable with the experimental values for various reasons. First, the $P_{b}$ center is split into two different occupation states that are not taken into account in this one-electron tightbinding model. Second, relaxation of the defect atom with a dangling bond will obviously modify the effective tight-binding parameters and hence the energy of the states. This variation could be introduced into the model by extending the impurity space, leading to an adjustment of the level position, but without any independent way of determining the parameters, it would have little physical meaning. In fact, the cluster calculations give a level for the stable relaxed configuration of the defect at the wrong position and there is an uncertainty of about $0.14 \AA$ on the position of the defect atom. ${ }^{7}$ Third, the value of the site energy of the defect atom could modify the energy of the level drastically. These effects are demonstrated by the results in Fig. 8. When $U_{H}$ at the defect orbital is reduced by $0.9 \mathrm{eV}$, the dangling-bond energy has become a

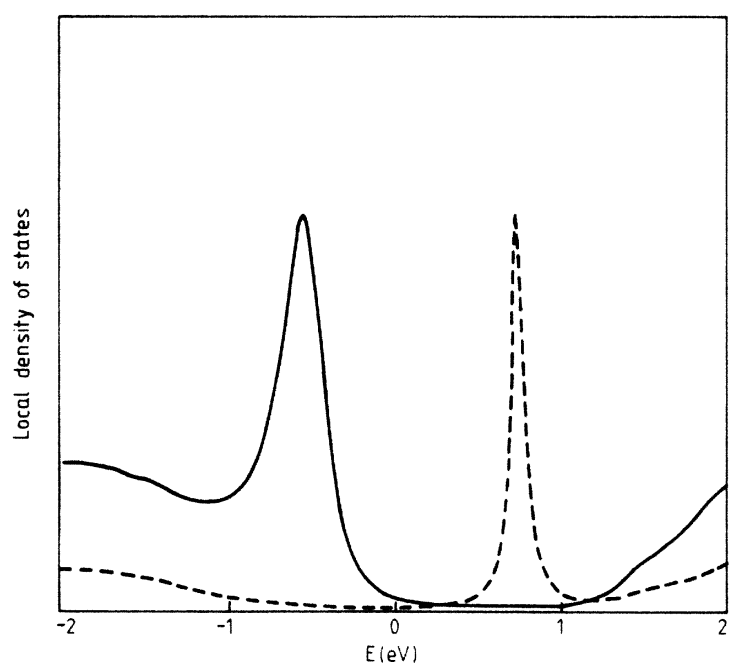

FIG. 8. Local density of states around the $\mathrm{Si}$ gap in a $\mathrm{Si}$ (111) $/ \mathrm{a}-\mathrm{SiO}_{2}$ interface; the resonance in the valence band is obtained when the site energy $U_{H}$ of the defect atom is decreased by $0.9 \mathrm{eV}$. Dashed curve denotes the same, but with $U_{H}$ increased by $1.2 \mathrm{eV}$.

resonance in the valence band. If $U_{H}$ is increased by 1.2 $\mathrm{eV}$ above the $\mathrm{Si}$ bulk value, the dangling-bond state is now in the gap with an energy increased by $\sim 0.6 \mathrm{eV}$. By a suitable choice of parameters it is clear that the danglingbond states corresponding to single and double occupation could be located in the gap, as observed experimentally.

In spite of all these problems, we can state that the important results obtained from this model are the following. (1) The $P_{b}$ center produces a state in the gap in the (110) and (111) interfaces, but not in the (100) case. If correct this will result in the improvement of the performance of devices that use (100) interfaces. (2) The presence of oxygen in the interface does not affect the dangling-bond local response in the (111) and (110) cases very much, but it is important in the (100) surface because there the defect atom is also attached directly to an oxygen. (3) A theory that calculates the site energy of the Si atom in different environments is essential in order to obtain quantitatively reliable energy levels for the $P_{b}$ center. Nevertheless, this does not invalidate the qualitative result since we have performed calculations varying the site energy between the clear surface and averaged interpolated values and, in any case, the energy of the $P_{b}$ center fluctuated by more than $10 \%$.

\section{ACKNOWLEDGMENTS}

We wish to thank W. B. Fowler and E. Poindexter for useful discussions. One of us (R.A.B.) is grateful to Oxford University for the hospitality received. This work was supported in part by the U.S. Army through its European Research Office. 
conductors, edited by J. F. Verweij and D. R. Walters (North-Holland, Amsterdam, 1983), p. 24.

${ }^{3}$ Y. Nishi, Jpn. J. Appl. Phys. 10, 52 (1971).

${ }^{4}$ E. H. Poindexter, P. J. Caplan, J. J. Finnegan, N. M. Johnson, D. K. Biegelsen, and M. D. Moyer, in The Physics of MOS Insulations, edited by G. Lucovsky, S. T. Pantelides, and F. L. Galeener (Pergamon, New York, 1980), p. 326.

${ }^{5}$ E. H. Poindexter and P. J. Caplan, Prog. Surf. Sci. 14, 197 (1983).

${ }^{6}$ T. Sakurai and T. Sukano, J. Appl. Phys. 52, 2889 (1981).

${ }^{7}$ A. H. Edwards, J. Electron. Mater. 14a, 491 (1985).

${ }^{8}$ R. J. Elliott, J. A. Krumhansl, and P. L. Leath, Rev. Mod. Phys. 46, 465 (1974).

${ }^{9}$ L. M. Falicov and F. Yńduráin, J. Phys. C 8, 1563 (1975).
10S. L. Cunningham, Phys. Rev. B 10, 4988 (1974).

${ }^{11}$ D. J. Chadi and M. L. Cohen, Phys. Status Solidi B 68, 405 (1975).

${ }^{12}$ P. Vogl, H. P. Hjalmarson, and J. D. Down, J. Phys. Chem. Solids 44, 365 (1983).

${ }^{13}$ K. C. Pandey and J. C. Phillips, Phys. Rev. Lett. 32, 1433 (1974).

${ }^{14}$ A. S. Carrico, Ph.D. thesis, Oxford University, 1985.

${ }^{15}$ R. B. Laughlin, J. D. Joannopoulos, and D. J. Chadi, Phys. Rev. B 21, 5733 (1980).

${ }^{16}$ E. Martinez and F. Yńduráin, Phys. Rev. B 25, 6511 (1984).

${ }^{17}$ R. A. Barrio, R. J. Elliott, and A. Carrico, following paper, Phys. Rev. B 34, 888 (1986).

${ }^{18}$ E. P. O'Reilly and J. Robertson, Phys. Rev. B 27, 3780 (1983). 